\title{
Comparing magnetic triggering of ELMs in TCV and ASDEX Upgrade
}

\author{
S H Kim ${ }^{1}$, M M Cavinato ${ }^{2}$, V Dokuka ${ }^{3}$, A A Ivanov ${ }^{4}$, \\ R R Khayrutdinov ${ }^{3}$, P T Lang ${ }^{5}$, J B Lister ${ }^{1}$, V E Lukash ${ }^{6}$, Y R Martin ${ }^{1}$, \\ S Yu Medvedev ${ }^{4}$ and L Villard ${ }^{1}$ \\ ${ }^{1}$ Centre de Recherches en Physique des Plasmas, Association EURATOM-Confédération Suisse, \\ EPFL, CH-1015 Lausanne, Switzerland \\ ${ }^{2}$ Consorzio RFX, Associazione Euratom-ENEA per la fusione, Padova, Italy \\ 3 TRINITI, Troitsk, Moscow Region, 142190, Russia \\ ${ }^{4}$ Keldysh Institute for Applied Mathematics, Russian Academy of Science, Moscow, Russia \\ ${ }^{5}$ Max-Planck-Institut für Plasmaphysik, EURATOM Association, Garching, Germany \\ ${ }^{6}$ Institute of Nuclear Fusion, RRC Kurchatov Institute, Moscow, 123182, Russia \\ E-mail: sunhee.kim@epfl.ch
}

Received 29 October 2008, in final form 6 February 2009

Published 15 April 2009

Online at stacks.iop.org/PPCF/51/055021

\begin{abstract}
Frequency locking of edge localized modes (ELMs) to the vertical plasma movements induced by magnetic perturbations first demonstrated in TCV was successfully repeated in ASDEX Upgrade. However, the ELMs were triggered in ASDEX Upgrade when the plasma was moving down towards the X-point with a consequent decrease in the plasma current density in the edge region, in contrast to the previous observation on TCV in which ELMs were triggered when the edge current was increased by an upward plasma movement. This opposite behaviour observed in the magnetic triggering of ELMs has been investigated by using a free-boundary tokamak simulator, DINA-CH. The passive stabilization loops (PSLs) located inside the vacuum vessel of ASDEX Upgrade produce similar external linking flux changes to those generated by the G-coil sets in TCV for opposite vertical plasma movements. Therefore, both plasmas experience similar local flux surface expansions near the upper G-coil set and PSL when the ELMs are triggered. In ASDEX Upgrade, however, the localized expansion of the plasma flux surfaces near the upper PSL is observed with the global shrinkage of the plasma column accompanied by the downward plasma movement.
\end{abstract}

(Some figures in this article are in colour only in the electronic version) 


\section{Introduction}

The high confinement mode (H-mode) observed in many tokamak plasmas is characterized by a pedestal region in which the plasma density and temperature profiles have strong radial gradients. While in the standard/baseline scenarios pressure gradients in the core are limited by micro-turbulent effects leading to profile stiffness, the pedestal pressure gradient is increased by a spontaneous formation of edge transport barriers, resulting in the localization of large bootstrap currents in the edge region. However, these continuous increases in the pressure gradient and the bootstrap current make the plasma edge susceptible to MHD instabilities. The onset of unstable MHD modes breaks the edge transport barriers and causes the plasma to release its stored energy and particles rapidly, in a few milliseconds. This fast repetitive regulation of the plasma energy and particle balances is known as edge localized modes (ELMs) and various types of ELMs have been identified in many tokamaks [1].

The ELMs, although degrading the plasma confinement, have some beneficial influences which allow quasi-stationary tokamak operation. Dangerous disruptive behaviour, such as an uncontrollable rise of plasma density or an accumulation of impurities in the plasma interior, is avoided by repetitive ELMs. This aspect made ITER consider an ELMy H-mode as its baseline operation. However, type-I ELMs anticipated during the main heating phase of ITER will produce unacceptably large heat loads on the plasma facing components, if the present scaling laws are extrapolated to ITER.

Although alternatives to the large type-I ELMs, such as grassy type-II ELMs and mixed type-I and type-II ELMs, are being studied, they still have very narrow operational windows $[2,3]$. Substantial progress has been made in the study of controlling the ELM frequency which is found to be inversely related to the heat load per ELM. Pellet injection into the pedestal region in ASDEX Upgrade [4], ergodization of magnetic field in DIII-D [5] and magnetic triggering of ELMs in TCV [6] were successful in modifying and controlling the ELM frequency. In particular, the magnetic triggering of ELMs is relatively easy to apply in a vertically elongated plasma, without the need for any additional systems.

In the TCV experiments, a pre-programmed voltage perturbation was injected on the G-coil sets located inside the vacuum vessel for the active control of the vertical instability [6]. The induced vertical plasma movements locked the ELM frequency to the magnetic perturbation by delaying and/or triggering ELMs. Similar experiments were carried out in ASDEX Upgrade by forcing the plasma to move vertically following an imposed reference waveform [7]. Both experiments were successful in triggering ELMs and controlling the ELM frequency. However, an unexpected and so far unexplained opposite behaviour was identified. In ASDEX, ELMs are triggered when the plasma is moving down towards the $\mathrm{X}$-point with a decrease in plasma current density in the edge region, in contrast to the TCV experiments in which ELMs are triggered when the plasma moves up with an increase in edge current density. Since the divertor current has the same sign as the plasma current, reducing their separation causes an increase in the flux linking the plasma, and vice versa. This linking flux is compensated by an inductively driven current at the plasma surface which has an opposite sign to the plasma current. Moving towards the X-point therefore always creates a negative induction by Lenz's law. In this paper, the sign of the plasma current is assumed to be positive for both tokamak plasmas.

This paper focuses on understanding this opposite behaviour observed in the magnetic triggering of ELMs and the possible physics reasons behind it. The geometries of TCV and ASDEX Upgrade used in our studies are shown in figure 1, including the separatrices of the plasmas studied in these simulations. Free-boundary features which have previously received little attention, such as plasma shape deformation, are investigated using the DINA-CH 

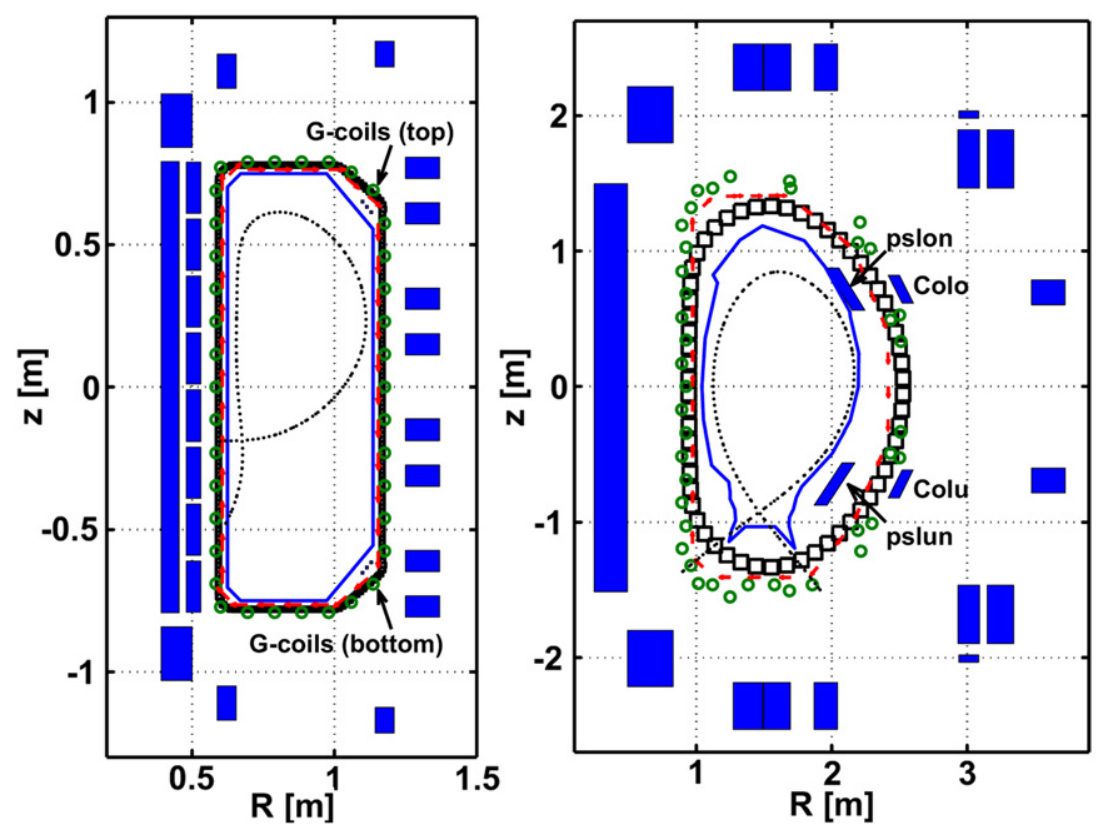

Figure 1. Definitions of TCV (left) and ASDEX Upgrade (right) used in the DINA-CH freeboundary tokamak simulations. The poloidal field coils (blue filled squares), vacuum vessels (black open squares), limiters (blue lines), separatrixes (black dots), flux loops (green circles) and magnetic probes (red arrows) are shown. The G-coil sets are located inside the vacuum vessel of TCV. The PSLs, pslon and pslun, and active control coils, CoIo and CoIu, are located inside and outside the vacuum vessel of ASDEX Upgrade, respectively.

free-boundary tokamak simulator [8]. Analysis of the kink-ballooning modes (toroidal mode numbers $n$ up to 60) possibly involved in ELM destabilization is provided by the KINX ideal MHD stability code [9].

In section 2 of this paper, the magnetic triggering of ELMs is simulated for TCV and ASDEX Upgrade. These simulations are compared in section 3, to examine possible causes of the observed opposite behaviour. Driving radial plasma movements as another possible technique of magnetic ELM triggering is studied in section 4. A discussion is presented in section 5 .

\section{Simulating the magnetic triggering of ELMs}

\subsection{Scope of the simulations}

Our major concern in this paper is to look for plausible mechanisms behind the opposite behaviour observed in the magnetic triggering of ELMs in two tokamaks. This paper does not model the ELMs themselves. The approach taken in this paper is simply to look for changes provoked by the magnetic perturbations which might reasonably be considered as candidates for influencing the ELM triggering conditions.

ELMs are not simulated in detail for two principal reasons. First, the cyclic ELM process is not yet completely understood. The onset conditions of ELMs and the transport processes of heat and particles across the pedestal region still have major uncertainties. Second, temporal variations of the pedestal current and pressure profiles during the ELM cycle can non-linearly 


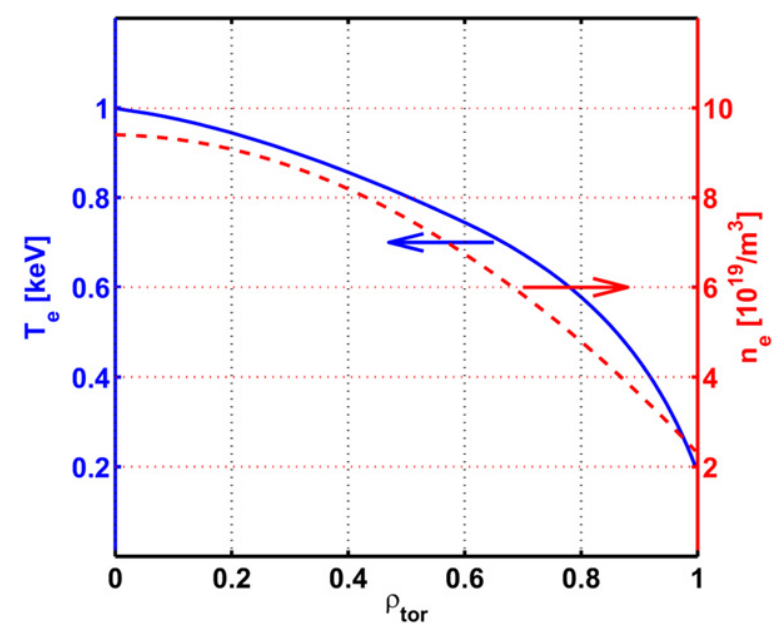

Figure 2. Prescribed monotonic electron temperature (blue solid line) and density (red dashed line) profiles used in the simulation of TCV discharge \#20333. These profiles are arbitrarily chosen to represent a typical H-mode TCV plasma.

interact with injected magnetic perturbations. This increases the complexity in the system being modelled and makes it very difficult to distinguish the influences contributed by the magnetic perturbations.

Including realistic pedestal profiles in the simulations was found not to be critical for investigating the dynamic plasma response, if the global plasma parameters, such as the total plasma current, internal inductance and poloidal plasma beta, are prescribed to be close to the values measured in the experiments. For convenience, the plasma density and temperature profiles are assumed to be monotonic from the plasma core to the separatix. The absence of a detailed description of the pedestal region and the resulting underestimated pressure gradient and bootstrap current in the edge region does not significantly change the free-boundary features of the plasma responses. This was verified by free-boundary simulations done by the PET code integrated into the DINA-CH Simulink environment with differently designed pedestal profiles and increased numerical resolution, which led to similar qualitative plasma responses [10].

\subsection{Magnetic triggering of ELMs in TCV}

TCV discharge \#20333 was chosen to investigate the magnetic triggering of ELMs in TCV. A comparison between this discharge and its first simulation was originally reported in detail in [6]. This discharge, in which the plasma is in a single-null lower configuration (SNL) with a magnetic centre shifted upward from the mid-plane $\left(z_{\text {mag }} \sim 0.2 \mathrm{~m}\right)$, shows successful ELM frequency locking to the vertical plasma movement induced by magnetic perturbations. In the simulation of this discharge, monotonic plasma density and temperature profiles representing typical H-mode core plasmas in TCV are prescribed as shown in figure 2. Transport of heat and particles is not modelled. The free-boundary plasma evolution is self-consistently calculated with the currents in the poloidal field coils and surrounding conducting systems using the DINA-CH tokamak simulator.

The plasma responses observed in the experiment [6] were successfully reproduced. The voltage applied to the G-coil sets $\left(V_{\mathrm{G}}\right)$ is perturbed by a short and strong signal input $\left(V_{\text {Pert }}\right)$. The perturbed current $\left(I_{\mathrm{G}}\right)$ flowing in G-coil sets induces a vertical plasma movement $(\Delta z$ and $\left.v_{z}\right)$ and results in a variation of the plasma current in the edge region $\left(\Delta I_{\text {edge, }}\right.$, which is an 


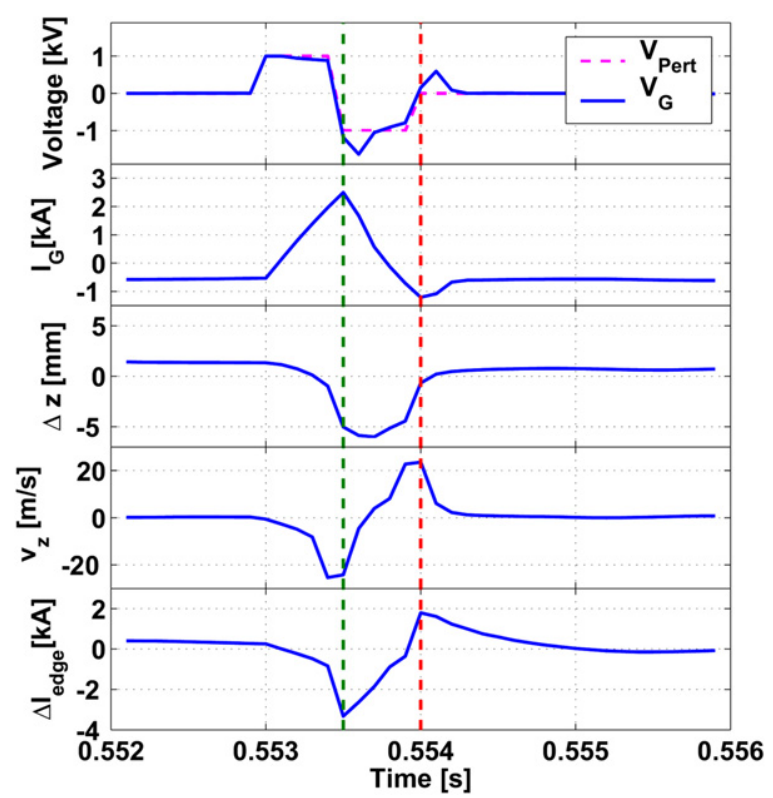

Figure 3. Magnetic triggering of ELMs in TCV discharge \#20333 is simulated. Time traces of the voltage and current in the G-coil sets, the vertical position of the magnetic centre, the velocity of the vertical movement and the variation of the edge current which is integrated outside $\rho_{\text {tor }}>0.95$ are shown. ELMs are triggered in the experiment when the plasma moved up with a consequent increase in edge current density (right-hand red dashed vertical line). The edge current density is correspondingly decreased with a downward plasma movement (left-hand green dashed vertical line).

integrated plasma current outside $\left.\rho_{\text {tor }}>0.95\right)$ as shown in figure 3. The plasma experiences repetitive vertical excursions of its magnetic centre $\left(z_{\mathrm{mag}}\right)$ of a few millimetres in response to G-coil current fluctuations of about $2 \mathrm{kA}$. The magnetic axis and the plasma centroid calculated taking the plasma current distribution into account show very similar responses. In the experiment, ELMs are triggered when the plasma moved up at the end of each magnetic perturbation.

The increase in edge current density resulting from positively induced currents due to an upward plasma movement away from the X-point was originally proposed as a candidate mechanism which triggers ELMs [6]. This was further emphasized by experiments in a single-null upper configuration (SNU), in which ELMs were triggered with downward plasma movements away from the X-point which again induce positive currents in the edge region. Besides the vertical plasma motion sweeping the up-down asymmetric vacuum field, there is another direct source of current. A net change in external flux linking the plasma, resulting from the proximity of the plasma column to one or other of the two G-coil sets, can drive current in the edge region. These drive a surface loop voltage $V_{\text {surf }}$ as given in [6] by

$$
V_{\text {surf }}=-\frac{\mathrm{d}}{\mathrm{d} t}\left\langle\psi_{\text {ext }}\right\rangle=-\frac{\partial}{\partial t}\left\langle\psi_{\text {ext }}\right\rangle-\left\langle\boldsymbol{u} \cdot \nabla \psi_{\text {ext }}\right\rangle=V_{\text {surf }}^{\text {direct }}+V_{\text {surf }}^{\text {motional }},
$$

where $\psi_{\text {ext }}$ is the external poloidal flux and the brackets represent averaging over the last closed flux surface. However, the voltage directly driven by the external source considering no plasma motion, $V_{\text {surf }}^{\text {direct }}$, is relatively small and has the opposite sign to the voltage induced by the vertical plasma motion sweeping the vacuum field, $V_{\text {surf }}^{\text {motional }}$. For example, if the plasma is in a SNL configuration close to the upper G-coil set in which current flows in the opposite 


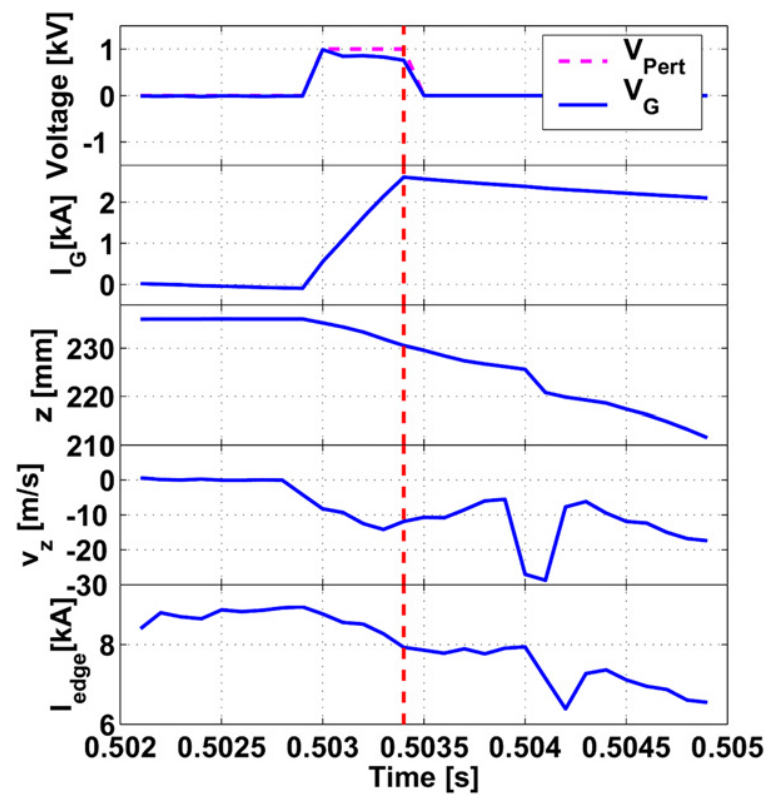

Figure 4. A downward VDE is triggered in TCV discharge \#20333 simulation. Time traces of the voltage and current of G-coil, the vertical position of magnetic centre, the velocity of the vertical movement and the variation of the edge current are shown. The feedback control systems are disabled for the plasma to continue the vertical displacement $\left(V_{\mathrm{G}}=0\right.$ after the red dashed vertical line).

direction to the plasma current, the plasma moves down and approaches the X-point, as the current in the G-coil sets is increased. This plasma movement induces negative currents in the edge region, while the net surface loop voltage drives a positive current compensating the decrease in external linking flux from the upper G-coil set to the plasma.

The perturbed edge current shown in figure 3 is approximately proportional to the velocity of the vertical plasma movement, again indicating that the current driven by the net surface loop voltage is relatively small. An additional simulation in which a vertical displacement event (VDE) was triggered by a pulsed magnetic perturbation and an immediate disabling of the control system is shown in figure 4 . The edge current evolution is clearly related to the velocity of the vertical plasma motion in the absence of a net surface loop voltage.

\subsection{Magnetic triggering of ELMs in ASDEX Upgrade}

The magnetic triggering of ELMs first demonstrated in TCV was repeated in ASDEX Upgrade [7]. Instead of injecting short and strong pulses into the coil systems, a reference vertical plasma position including a sinusoidal waveform is pre-programmed in the feedback control system to produce the magnetic perturbations. ASDEX Upgrade discharge \#18343 showing the ELM frequency locking to the vertical plasma motion [7] is chosen for a freeboundary tokamak simulation. The plasma density and temperature profiles are prescribed with monotonic shapes as shown in figure 5 . The choice of the temperature profile is made during the initialization of a simulation and is constrained to have a similar shape to the data points. The assumed profiles show some deviations from the data points, since the initial temperature profile has to be consistent with the measured plasma pressure (through $\beta_{\mathrm{p}}$ ) and the assumed density profile. 


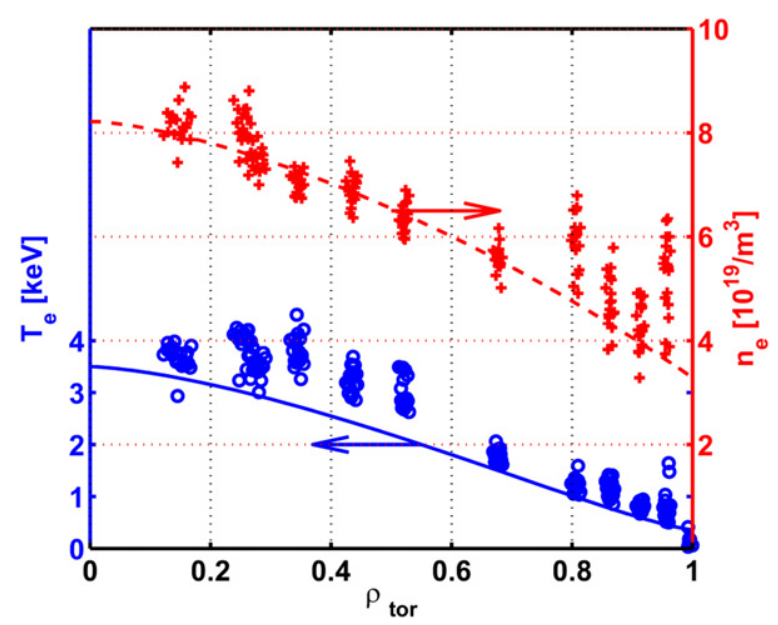

Figure 5. Prescribed monotonic electron temperature (blue solid line) and density (red dashed line) profile used in the simulation of ASDEX Upgrade discharge \#18343. These profiles are chosen based on the measured data. Blue circles and red crosses indicate the measured electron temperature and density, respectively.

Simulated plasma responses are shown in figure 6. The pre-programmed reference input $\left(z_{\text {ref }}\right)$ to the feedback control system generates currents in the active control coils (CoIo and $\mathrm{CoIu})$ for the control of the vertical plasma position $\left(z_{\mathrm{mag}}\right)$. Eddy currents are induced in the vacuum vessel and passive stabilization loops (PSLs, pslon and pslun), in response to the controlled coil currents and plasma movement. The variation of the plasma current in the edge region $\left(\Delta I_{\text {edge }}\right)$ is again a mixture of motional $\left(v_{z}\right)$ and direct induction contributions (equation (1)). $I_{85}$ and $I_{95}$ represent the edge currents integrated outside $\rho_{\text {tor }}>0.85$ and $\rho_{\text {tor }}>0.95$, respectively. The times the plasma is moved up and down are indicated by the left-hand (green) and right-hand (red) dashed vertical lines, respectively.

The controlled vertical plasma position responds with a larger phase delay with respect to the reference waveform in the simulation $(\sim 3 \pi / 2)$ than that observed in the experiment [7] $(\sim \pi)$. However, key components representing the dynamic plasma response, such as the magnitude of the vertical excursion $\left(\Delta z_{\mathrm{mag}} \sim \pm 0.7 \mathrm{~cm}\right)$ and its velocity $\left(v_{z} \sim \pm 3 \mathrm{~m} \mathrm{~s}^{-1}\right)$, are reproduced similar to the observations in the experiment. The peaks shown in the velocity of the vertical movement originate from a large mesh size used in determining the plasma centre. The edge current $\left(I_{95}\right)$ is perturbed mainly by the vertical plasma motion, deduced from the similarity of their evolutions. However, comparing the edge currents, $I_{85}$ and $I_{95}$, we see that the phase difference with respect to the plasma motion varies with the integration range. When the plasma is moving down, the edge plasma current is reduced by negatively induced currents, and vice versa. In spite of the complexity of the simulated system, this matches well with the observations in the experiments.

The peak to peak variation of $\Delta I_{\text {edge }}(\sim 7 \mathrm{kA})$ is considerably lower than the swing ( $\sim 50-60 \mathrm{kA})$ shown in figure 4 of [7]. This difference can be attributed to the lower edge temperature used in the simulation (see figure 4 ). The edge current variation is significantly reduced by the resulting lower plasma conductivity and is further reduced by a slightly lower surface voltage induced by a smaller vertical movement. The lower edge temperature and the absence of pedestal-like edge profiles also reduce the edge bootstrap current which provides the main contribution to the average edge current. Although the absolute value appears to be 


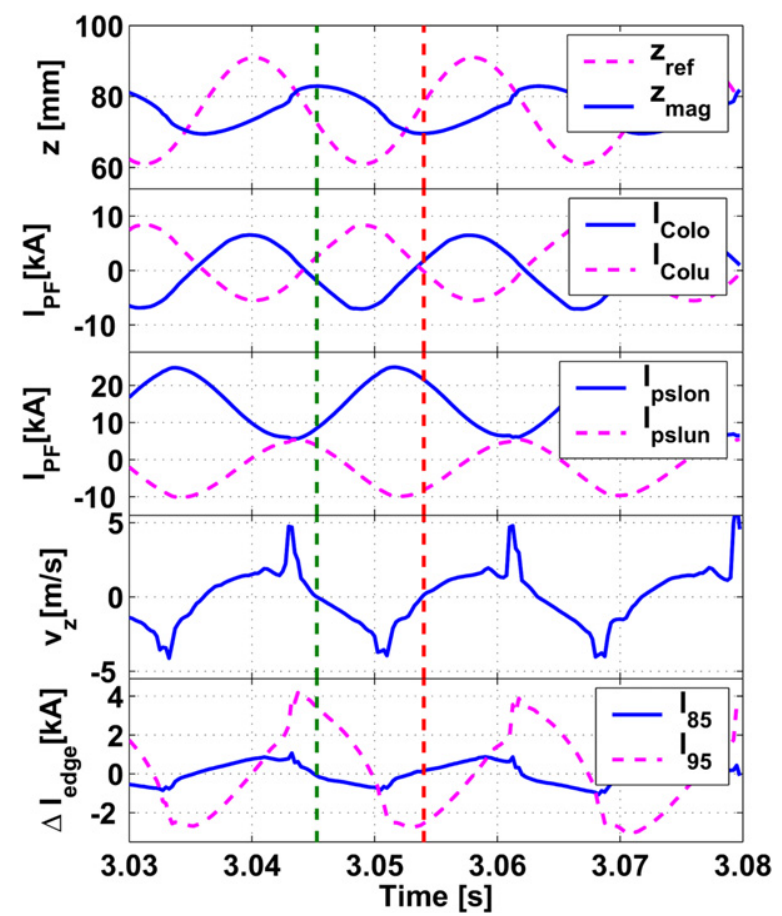

Figure 6. Magnetic triggering of ELMs in ASDEX Upgrade discharge \#18343 is simulated. Time traces of the reference and controlled vertical positions, the currents in the fast vertical position control coils ( $I_{\mathrm{CoIo}}$ and $\left.I_{\mathrm{CoIu}}\right)$, the currents in the PSLs $\left(I_{\mathrm{pslon}}\right.$ and $\left.I_{\mathrm{pslun}}\right)$, the velocity of the vertical plasma movement and the variations of edge current densities. $I_{85}$ and $I_{95}$ represent integrated currents outside $\rho_{\text {tor }}>0.85$ and $\rho_{\text {tor }}>0.95$, respectively. ELMs are triggered in the experiments when the plasma is moving down at maximum speed (between the dashed vertical lines) and the edge current density is decreased.

quite far from the experiment, the normalized edge current variation with respect to the average edge current, which defines the relative strength of the edge current perturbation, is similar for the simulation and the experiment.

\section{Comparing the two cases of magnetic triggering of ELMs}

The type of ELM observed in the magnetic triggering experiments in TCV has recently been identified as type-III [11], whereas type-I ELMs are observed during non-triggering phases in ASDEX Upgrade. This implies that the magnetic perturbations injected into TCV and ASDEX Upgrade discharges might be triggering ELMs in different ways. Different ELM types imply different plasma sensitivities to the dynamic changes of physical quantities such as the edge pressure gradient and plasma current density. The opposite behaviour observed in the magnetic triggering of ELMs might then require different explanations. However, this issue is beyond our current modelling capability which does not yet simulate the ELMs themselves and therefore cannot differentiate between different ELM types.

In the occurrence of natural ELMs, the pedestal pressure gradient increases with a build-up of bootstrap current, until reaching a threshold value, and then it decreases quickly releasing the plasma particles and stored energy. This threshold behaviour is generally believed to be caused by destabilizing MHD modes with the increase in pressure gradient and current 

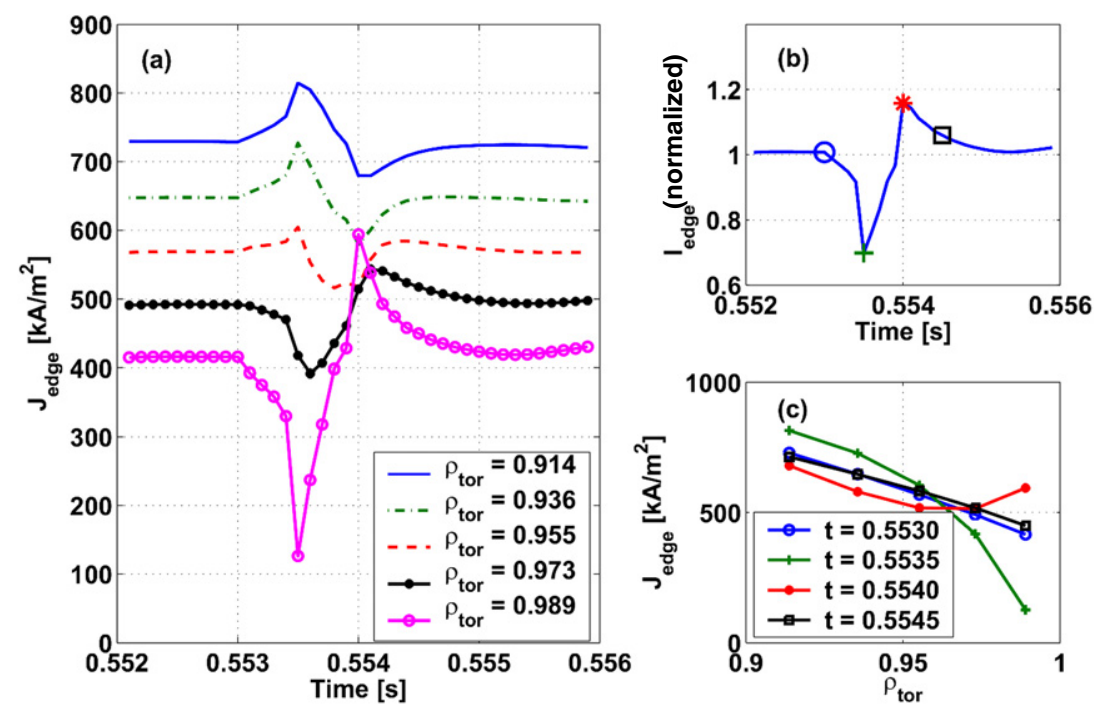

Figure 7. Evolution of the plasma current in the simulation of TCV discharge \#20333. Edge current density $(a)$, normalized edge current integrated outside $\rho_{\text {tor }}>0.95(b)$ and radial edge current density profiles $(c)$ at the times indicated by the markers in $(b)$ are shown.

density in the edge region. If the edge pressure gradient and/or the edge current density are/is significantly perturbed externally, the edge stability and therefore the ELM cycle would clearly be modified. This can either delay or trigger ELMs, leading to synchronization of the ELMs to the perturbation.

\subsection{Perturbed plasma current density in the edge region}

The evolution of the simulated plasma current density in the edge region is shown in figures $7(a)$ and $8(a)$, respectively for TCV and ASDEX Upgrade. A significant feature is observed in the evolution of the plasma current density. When the plasma current density is reduced by negatively induced currents at the plasma boundary, the plasma current density at the inner magnetic flux surfaces is increased, and vice versa. In both plasmas, the depth of the skin current calculated with a given edge temperature is comparable to the distance between the magnetic flux surface of $\rho_{\text {tor }}=0.9$ and the plasma boundary. This is the range in which the edge current perturbations are observed. The current diffusion time calculated with this scale length is less than the period of the magnetic perturbation in both plasmas. Therefore, the perturbed current penetrating the edge region and its radial diffusion across flux surfaces produces a complicated pattern of the edge current fluctuation.

Edge current density profiles plotted on the radial grid are given in figures $7(c)$ and $8(c)$ at the moments marked in figures $7(b)$ and $8(b)$, respectively. They clearly show the inverted perturbations. The edge currents integrated outside $\rho_{\text {tor }}>0.95$ are normalized with averaged unperturbed edge plasma currents and plotted in figures $7(b)$ and $8(b)$. The $\mathrm{min} / \mathrm{max}$ variation of the normalized edge current in TCV is about 0.5 (from 0.7 to 1.2), while it is about 0.25 (from 0.9 to 1.15) in ASDEX Upgrade.

The large perturbation in edge current observed in TCV is mainly due to its faster vertical plasma movement $\left( \pm 20 \mathrm{~m} \mathrm{~s}^{-1}\right)$. In ASDEX Upgrade, although the speed of the vertical plasma movement $\left( \pm 3 \mathrm{~m} \mathrm{~s}^{-1}\right)$ is relatively low, it produces a large perturbation due to its 

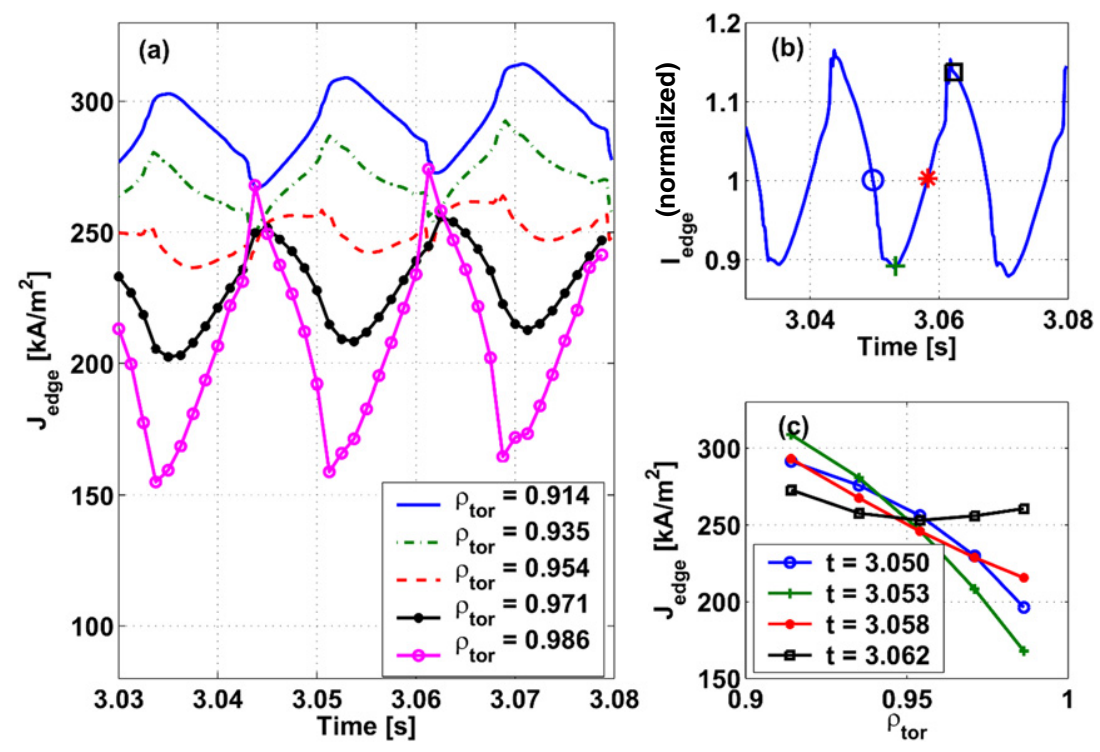

Figure 8. Evolution of the plasma current in the simulation of ASDEX Upgrade discharge \#18343. Edge current density $(a)$, normalized edge current integrated outside $\rho_{\text {tor }}>0.95(b)$ and radial edge current density profiles $(c)$ at the times indicated by the markers in $(b)$ are shown.

higher edge temperature. However, triggered ELMs in the ASDEX Upgrade experiments are observed when the edge current is reduced by negatively induced currents. Although there is a controversy over the destabilizing effect of the edge current gradient increase, the edge current reduction generally has a strong stabilizing effect. Therefore, a simple explanation based on the sign of the induced edge current perturbation is inadequate to explain both the magnetic triggering of ELMs in ASDEX Upgrade and the observed opposite ELM behaviour between two plasmas. In ASDEX Upgrade, we should therefore explore the possibility that ELMs might be triggered by another perturbed physical quantity.

\subsection{Perturbed local plasma pressure gradient in the edge region}

The response of the plasma to the magnetic perturbation not only induces a plasma motion but also produces a deformation of the plasma shape. The latter results in a perturbation of the local plasma pressure gradient in the edge region, as shown in figure 9. Assuming a non-variant pressure difference between two neighbouring magnetic flux surfaces, the variation of the local pressure gradient is calculated from the flux surface separation along the poloidal angle. This is given by

$$
\left.\frac{\Delta\left(\mathrm{d} p /\left.\mathrm{d} r\right|_{\theta}\right)}{\mathrm{d} p /\left.\mathrm{d} r\right|_{\theta}} \sim \frac{x_{0}-x_{1}}{x_{1}}\right|_{\theta},
$$

where $x_{0}$ and $x_{1}$ are the distances between two neighbouring magnetic flux surfaces at the beginning and at the end of an upward or downward vertical movement.

The variations are less than $10 \%$ of the unperturbed averaged value. In both TCV and ASDEX Upgrade, an increase in the local pressure gradient is produced by a downward plasma movement. In a SNL configuration, both plasmas are shrinking when they are moving down closer to the X-point, and vice versa. Therefore, although the increase in the local pressure gradient is destabilizing the edge in ASDEX Upgrade, it cannot be an answer for 

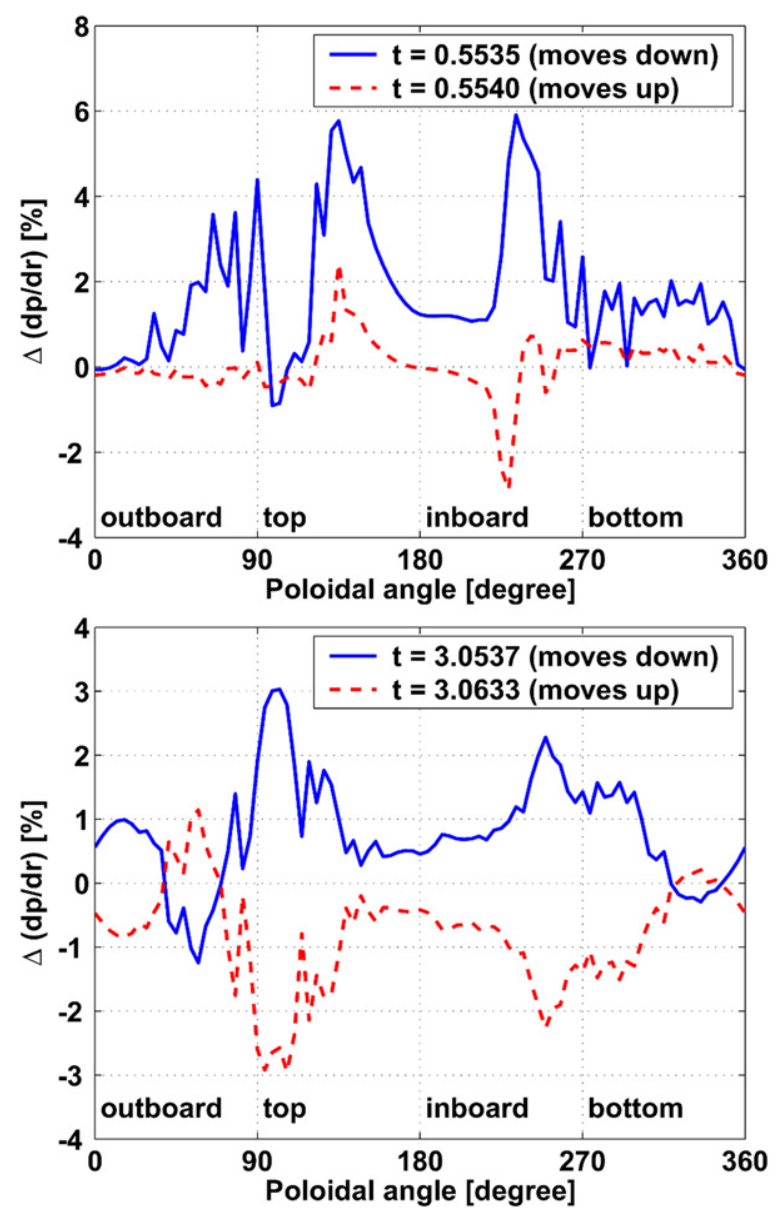

Figure 9. Local pressure gradient change in the edge region is calculated as a function of the poloidal angle. Downward (solid blue lines) and upward (red dashed lines) movements in TCV discharge \#20333 (top) and ASDEX Upgrade discharge \#18343 (bottom) are compared.

the observed opposite ELM behaviour between the two devices. In particular, the upward movement associated with the magnetic triggering of ELMs in TCV reduces the local pressure gradient in the edge region.

\subsection{Flux surface deformation and its pattern in the edge region}

An interesting feature is observed in the deformation of the plasma shape in ASDEX Upgrade. The plasma experiences a localized expansion of the flux surfaces near the upper PSL during its downward movement. In TCV, a similar flux surface expansion near the upper G-coil set is produced with an upward movement. These are compared in figure 10. The PSLs located inside the vacuum vessel of ASDEX Upgrade play a similar role to that of the G-coil sets in TCV due to their proximity to the plasma boundary. Positive eddy currents are induced in the upper PSL as a result of the downward plasma movement and direct induction by currents in active and passive conducting structures. They create a localized expansion of the flux surfaces near the upper PSL compared with the overall shrinkage of the plasma column. 

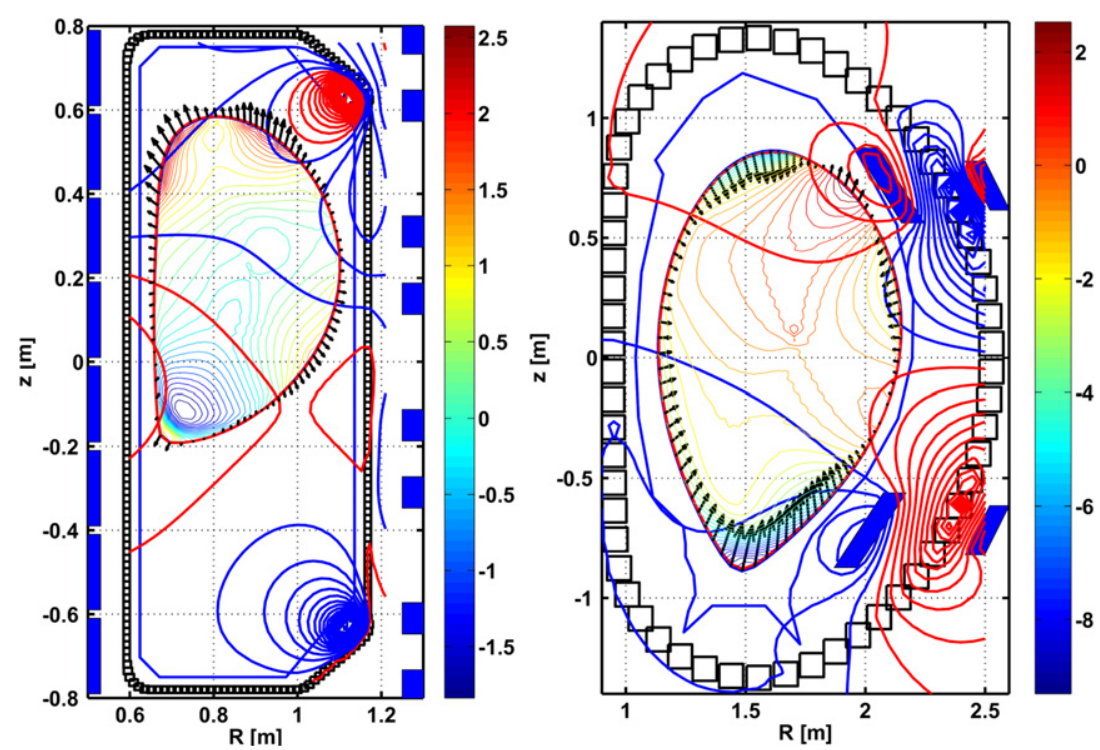

Figure 10. Flux surface deformations and vacuum flux changes are shown for upward plasma movement in TCV discharge \#20333 (left) and downward plasma movement in ASDEX Upgrade discharge \#18343 (right). ELMs are triggered in the experiments for these plasma movements. The arrows are amplified by a factor 20 for visibility.

Both plasma movements generating a localized expansion of flux surfaces, upwards in TCV and downwards in ASDEX Upgrade, trigger ELMs in the experiments. The opposite plasma movement, downwards in TCV and upwards in ASDEX Upgrade, creates localized shrinkage of flux surfaces as shown in figure 11. In these figures, the deformation of the plasma flux surfaces is calculated by using a moving frame in which the origin is fixed to the magnetic axis. The arrows, magnified 20 times for visibility, represent the deformation of the last closed flux surface. The increase and decrease in the external linking fluxes are shown in red and blue, respectively.

The responses of plasma shape parameters to the magnetic perturbations are shown in figure 12. The red dashed vertical lines indicate the times ELMs are triggered in the experiments $[6,7]$. In both plasmas, the plasma elongation is slightly delayed with respect to the vertical plasma movement and the size of the elongation perturbations is less than $1 \%$. The plasma triangularities respond promptly and exhibit a few percent variation. These global parameters do not reveal any additional clue for the opposite behaviour observed in the magnetic triggering of ELMs. However, a stability study on the ASDEX Upgrade plasmas, including the separatrices, showed that the increase in the plasma squareness in the low field side (LFS) and upper half plane of the poloidal cross section has a tendency to destabilize the plasma edge [10]. This tendency matches well with the localized expansion of flux surfaces near the upper PSL during the downward plasma movement in ASDEX Upgrade.

Although the localized expansion of flux surfaces observed both in the simulation of TCV and ASDEX Upgrade plasmas provides a new clue for understanding the magnetic triggering of ELMs, the shape deformation in TCV plasmas still has some open questions. DINA$\mathrm{CH} / \mathrm{SPIDER}$ simulations dedicated to the study of edge stability of TCV plasma during the magnetic triggering of ELMs [12] show that the plasma squareness in the LFS and upper half plane of the poloidal cross section decreases a little during the upward plasma movement resulting in a small stabilizing effect. 

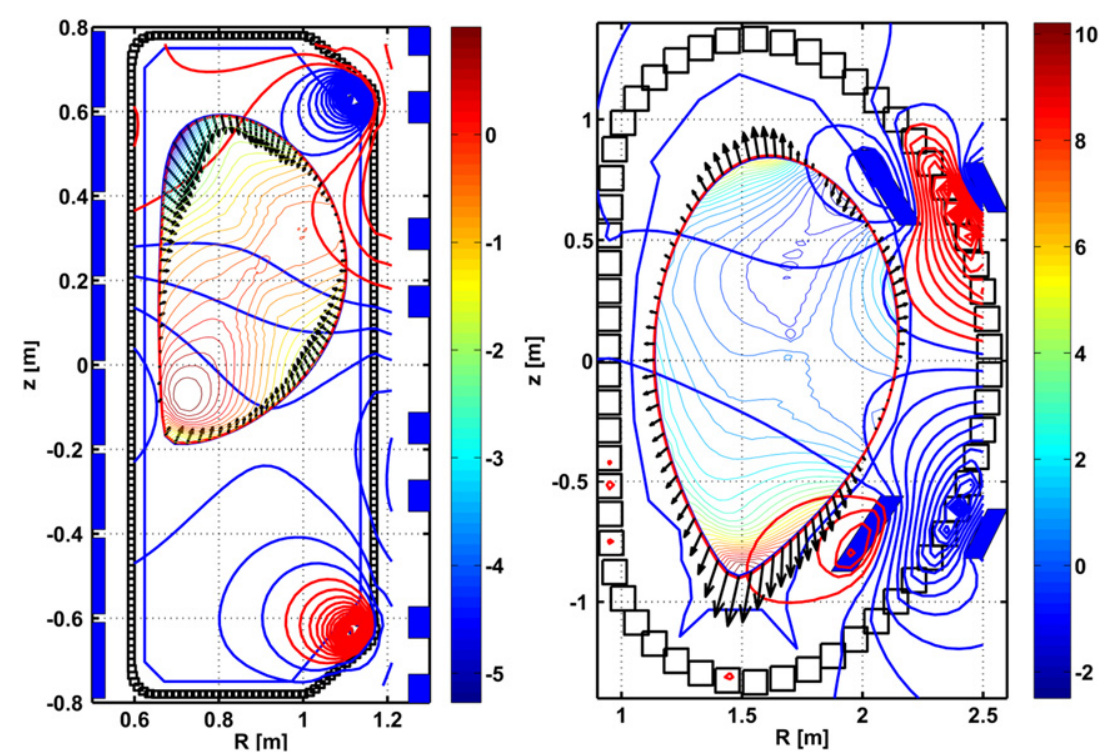

Figure 11. Flux surface deformations and vacuum flux changes are shown for downward plasma movement in TCV discharge \#20333 (left) and upward plasma movement in ASDEX Upgrade discharge \#18343 (right). ELMs are not observed in the experiments for these plasma movements. The arrows are amplified by a factor 20 for visibility.
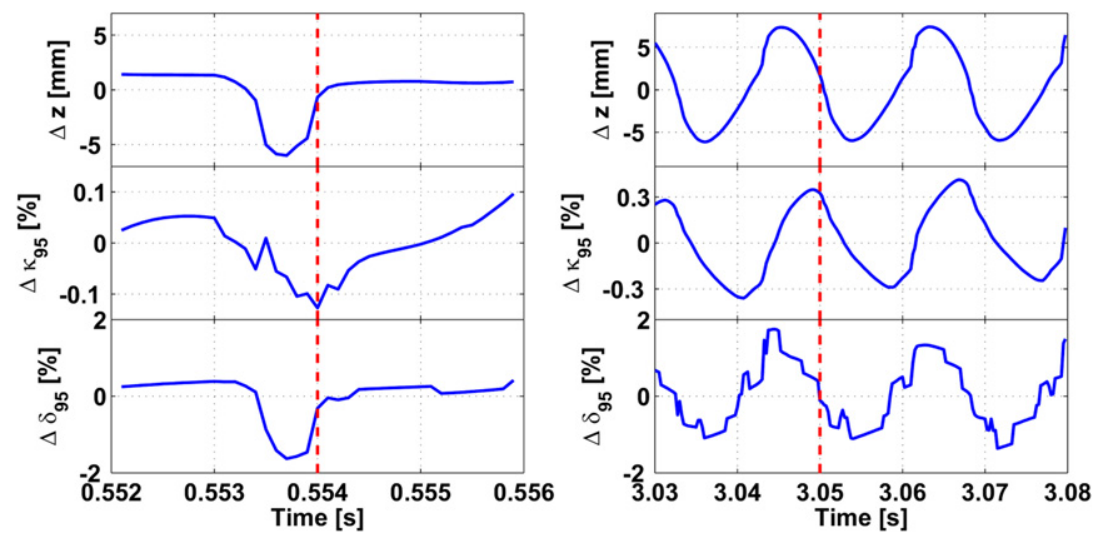

Figure 12. Vertical plasma movement and the response of the plasma elongation and triangularity to the magnetic perturbation are shown for TCV discharge \#20333 (left) and ASDEX Upgrade discharge \#18343 (right). The times ELMs are triggered in the experiment are indicated by the red dashed vertical lines.

\section{Magnetic perturbation with radial plasma movements in ASDEX Upgrade}

The effect of the plasma shape deformation was investigated further by minimizing fluctuations in the edge plasma currents. Instead of inducing a vertical plasma motion, a radial motion is induced to produce a different type of magnetic perturbation in the simulation of ASDEX Upgrade discharge \#18343. To avoid saturation of the input voltages to the active coils, the amplitude of the reference waveform modulation was reduced to half of that used to control the vertical plasma position. 

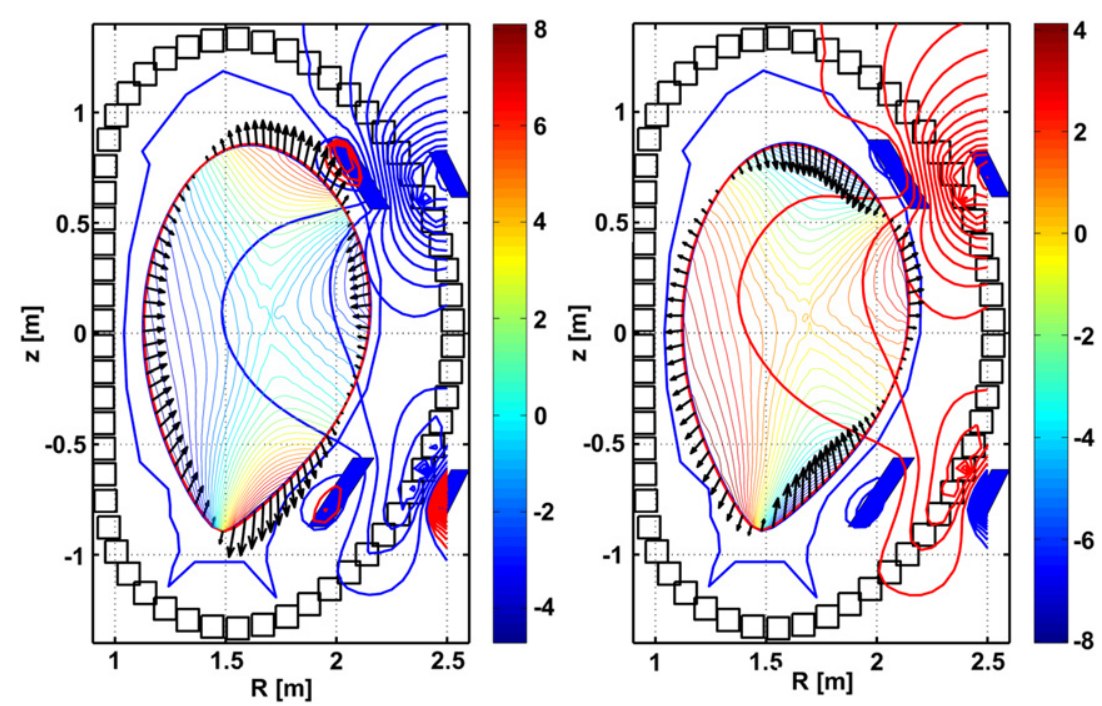

Figure 13. Flux surface deformations and vacuum flux changes are shown for inward (left) and outward (right) radial plasma movements in ASDEX Upgrade. The arrows are amplified by a factor 20 for visibility.

The plasma shape is easily deformed by a radial plasma movement due to the eddy currents induced in the PSLs and vacuum vessel as shown in figure 13. The deformation of the plasma shape shows a weak change in squareness in the LFS and upper half plane. The plasma elongation is the dominant parameter linked to the plasma shape deformation.

The deformation of the plasma boundary during vertical and radial plasma movements is compared in figure 14. The deformation patterns which accompany the radial movement (poloidal mode number, $m=2$ ) are simpler than those which accompany the vertical movement $(m=3)$ and the area of the plasma column is changed less, compared with the case of vertical plasma movement. In particular, the strongly localized expansion of the flux surface near the upper PSL against the shrinkage of the plasma column is observed only with the downward plasma movement, as indicated by blue circles.

The experiments reported in ASDEX Upgrade showed that the plasma shape deformation produced by a radial plasma movement does not trigger ELMs [13], and this observation is supported by a stability analysis with the KINX code [14]. The detailed analysis of the plasma boundary curvature perturbation shows similar changes for vertical and radial movement in the LFS and upper half plane. However, there is a difference affecting the edge stability in ASDEX Upgrade. The plasma boundary curvature in the LFS and lower half plane is increased only when the plasma column size is reduced by the downward plasma movement.

\section{Discussion}

The experimental evidence for ELM triggering from TCV and ASDEX Upgrade is clear and has been subsequently confirmed on JET [15]. The original explanation by TCV was an inductive increase in the edge current due to the plasma movement away from the divertor current. The observations on ASDEX Upgrade and JET confirmed that the original prediction that the effect would become clearer on larger plasmas with higher edge 

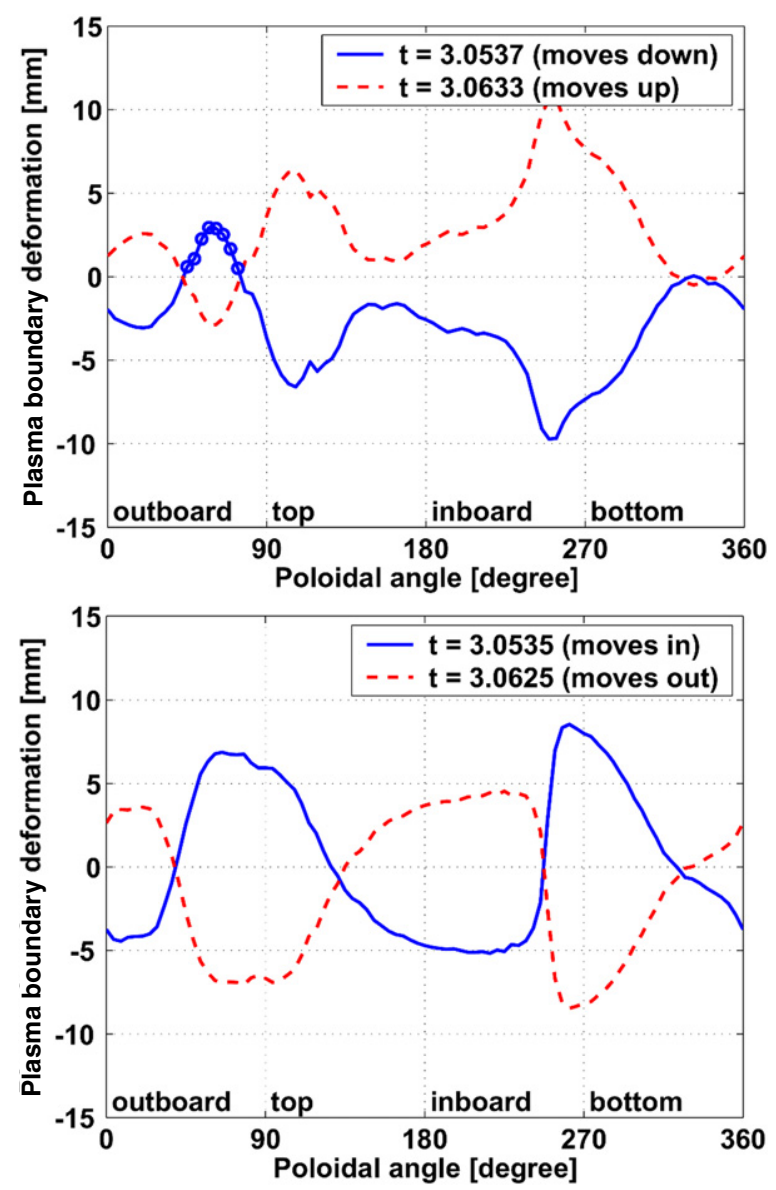

Figure 14. Plasma boundary deformations for vertical movement (top) and for radial movement (bottom) in ASDEX Upgrade. Localized expansion of the flux surfaces near the upper PSL is indicated (blue circles).

temperatures was correct. However, the change in sign of the edge current density change indicated that the simplicity of the original TCV explanation is probably inadequate. In this paper we have explored the physics of this phenomenon with two advanced codes, namely a free-boundary evolution code, DINA- $\mathrm{CH}$, and a free-boundary stability code including the separatrix, KINX. The qualitative observations obtained with these two models are summarized in table 1.

The preceding discussions on the various effects investigated have not clearly defined a unique mechanism for destabilizing the ELMs. While the edge current increase by an upward vertical movement is the strongest candidate in TCV, in ASDEX Upgrade the onset of ELMs seems to depend not only on the equilibrium quantities such as the edge pressure and current gradients but also on free-boundary motion and deformation. The role of the PSL in changing the separatrix shape during fast transients has been exposed. Localized expansion of the flux surfaces near the upper PSL in ASDEX Upgrade, compared with the overall shrinkage of the plasma column accompanied by the downward plasma movement, shows a tendency to destabilize the plasma edge in the KINX stability analysis. The perturbation induced by a 
Table 1. The observations in the experiments and simulations of magnetic triggering of ELMs are summarized.

\begin{tabular}{|c|c|c|c|c|}
\hline Observations & TCV & ASDEX & Jpgrade & Comments \\
\hline Plasma movement & Upward & Downward & Inward & \\
\hline Type of natural ELMs & Type III & Type I & Type I & \\
\hline Triggered ELMs & Observed & Observed & Not observed & \\
\hline Edge current density & Increased $^{\mathrm{a}}$ & Decreased & - & \\
\hline Edge current gradient & $\begin{array}{l}\text { Decreased } \\
\text { (locally } \\
\text { increased) }\end{array}$ & $\begin{array}{l}\text { Increased } \\
\text { (locally } \\
\text { decreased) }\end{array}$ & - & Mixed contributions \\
\hline Edge pressure gradient & Decreased & Increased $^{\mathrm{a}}$ & - & \\
\hline Plasma area & Expanded & Shrunken $^{\mathrm{a}}$ & - & \\
\hline Shape deformation & $\begin{array}{l}\text { Locally expanded } \\
\text { in upper LFS }\end{array}$ & $\begin{array}{l}\text { Locally expanded } \\
\text { in upper LFS }\end{array}$ & Elongated & \\
\hline Squareness (upper LFS) ${ }^{b}$ & Decreased & Increased $^{\mathrm{a}}$ & - & $\begin{array}{l}\text { Squareness decrease } \\
\text { in TCV is not yet } \\
\text { clearly explained }\end{array}$ \\
\hline Curvature (upper LFS) ${ }^{\mathrm{b}}$ & - & $\begin{array}{l}\text { Locally increased } \\
\text { or decreased }\end{array}$ & $\begin{array}{l}\text { Locally increased } \\
\text { or decreased }\end{array}$ & Similar patterns \\
\hline Curvature (lower LFS) ${ }^{\mathrm{b}}$ & - & Increased $^{\mathrm{a}}$ & Decreased & $\begin{array}{l}\text { Systematic differences } \\
\text { in the stability } \\
\text { margin behaviour }\end{array}$ \\
\hline
\end{tabular}

a Possible candidates for triggering ELMs.

b Observations from the KINX analysis [10, 14].

radial plasma movement, such as a strong elongation change, seems insufficient to trigger ELMs by itself.

The explorations conducted in this paper have underlined the difficulty in attributing a complex phenomenon to a single effect, since for a single given cause, namely fast stimulation of an equilibrium change, all potentially relevant mechanisms are excited, namely changes to the spatial pressure and current gradients, to the edge current density (averaged and local), to the boundary shape, to the plasma column size and to its position. Since all these effects are demonstrably linked to MHD instability, there is a worrying possibility of erroneously attributing causality in the presence of all correlated effects. Furthermore, since such fast effects are intimately linked to the physical construction of the passive and active conductors, with possible 3-D effects not excludable, there is even a strong possibility that similar plasmas in different tokamaks might behave differently, underlining the risk of extrapolating the results from these three experiments to ITER. However, the fact remains that the phenomenon of magnetic triggering might offer a control mechanism for ELMs in ITER, with no additional infrastructure required since internal coils have already been added.

\section{Acknowledgments}

This work, supported by the European Communities under the contract of Association between EURATOM/Confédération Suisse, and partly by the Fonds National Suisse de la Recherche Scientifique, was carried out within the framework of the European Fusion Development Agreement. The views and opinions expressed herein do not necessarily reflect those of the European Commission. 


\section{References}

[1] Zohm H 1996 Plasma Phys. Control. Fusion 38105

[2] Stober J, Marascheck M, Conway G D, Gruber O, Herrmann A, Sips A C C, Treutterer W, Zohm H and ASDEX Upgrade team 2001 Nucl. Fusion 411123

[3] Saibene G et al 2002 Plasma Phys. Control. Fusion 441769

[4] Lang P T et al 2004 Nucl. Fusion 44665

[5] Evans T E et al 2005 J. Nucl. Mater. 337-339 691

[6] Degeling A W, Martin Y R, Lister J B, Villard L, Dokouka V N, Lukash V E and Khayrutdinov R R 2003 Plasma Phys. Control. Fusion 451637

[7] Lang P T et al 2004 Plasma Phys. Control. Fusion 46 L31

[8] Favez J-Y, Khayrutdinov R R, Lister J B and Lukash V E 2002 Plasma Phys. Control. Fusion 44171

[9] Degtyarev L, Martynov A, Medvedev S, Troyon F, Villard L and Gruber R 1997 Comput. Phys. Commun. 10310

[10] Medvedev S Yu, Ivanov A A, Martynov A A, Poshekhonov Yu Yu, Kim S H, Lister J B, Martin Y R, Sauter O, Villard L and Khayrutdinov R R 2005 Proc. 32nd EPS Conf. on Plasma Physics (Tarragona, Spain) vol 29C (ECA) p P-5.064

[11] Behn R, Alfier A, Medevedev S Yu, Zhuang Ge, Pasqualotto R, Nielsen P, Martin Y and the TCV team 2007 Plasma Phys. Control. Fusion 491289

[12] Medvedev S Yu et al 2006 Proc. 33rd EPS Conf. on Plasma Physics (Rome, Italy) vol 30I (ECA) p P-1.146

[13] Lang P T et al 2006 Czech. J. Phys. 561329

[14] Medvedev S Yu, Ivanov A A, Martynov A A, Poshekhonov Yu Yu, Kim S H, Lister J B, Martin Y R, Sauter O, Villard L and Lang P T 2007 Proc. 34th EPS Conf. on Plasma Physics (Warsaw, Poland) vol 31F (ECA) p P-4.078

[15] Sartori F, Piccolo F, Zedda M K and JET-EFDA contributors 2008 Proc. 35th EPS Conf. on Plasma Physics (Hersonissos, Greece) vol 32D (ECA) p P-5.045 